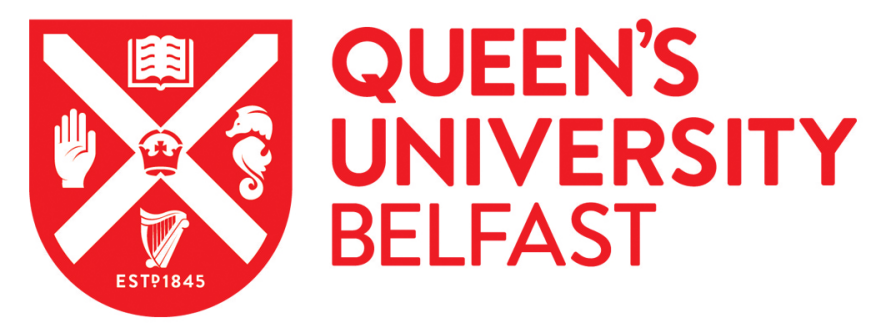

\title{
Modelling Asymmetric Conditional Dependence between Shanghai and Hong Kong Stock Markets
}

Wu, W., Lau, M. C. K., \& Vigne, S. A. (2017). Modelling Asymmetric Conditional Dependence between Shanghai and Hong Kong Stock Markets. Research in International Business and Finance, 1137-1149.

https://doi.org/10.1016/j.ribaf.2017.07.050

Published in:

Research in International Business and Finance

Document Version:

Peer reviewed version

Queen's University Belfast - Research Portal:

Link to publication record in Queen's University Belfast Research Portal

\section{Publisher rights}

Copyright 2017 Elsevier.

This manuscript is distributed under a Creative Commons Attribution-NonCommercial-NoDerivs License

(https://creativecommons.org/licenses/by-nc-nd/4.0/), which permits distribution and reproduction for non-commercial purposes, provided the author and source are cited.

\section{General rights}

Copyright for the publications made accessible via the Queen's University Belfast Research Portal is retained by the author(s) and / or other copyright owners and it is a condition of accessing these publications that users recognise and abide by the legal requirements associated with these rights.

Take down policy

The Research Portal is Queen's institutional repository that provides access to Queen's research output. Every effort has been made to ensure that content in the Research Portal does not infringe any person's rights, or applicable UK laws. If you discover content in the Research Portal that you believe breaches copyright or violates any law, please contact openaccess@qub.ac.uk. 


\title{
Modelling Asymmetric Conditional Dependence between Shanghai and Hong Kong Stock Markets
}

\begin{abstract}
This paper investigates the asymmetric conditional dependence between Shanghai and Hong Kong stock index returns, to assess the impact of the recent financial recession on Chinese equity markets using the Copula approach. We first propose methods for optimal model selection when constructing the conditional margins. The joint conditional distribution is then modeled by the timevarying copula, where the generalised autoregressive score (GAS) model of Creal, et al. (2013) is used to capture the evolution of the copula parameters. Upper and lower parts of the bivariate tail are estimated separately in order to capture the asymmetric property. We find the conditional dependence between the two markets is strongly timevarying. While the correlation decreased before the crisis, it increased significantly prior to 2008, pointing to the existence of contagion between the two markets. Moreover, we find a slightly stronger bivariate upper tail, suggesting the conditional dependence of stock returns is more significantly influenced by positive shocks in China. This finding is further confirmed by a test for asymmetry which shows that the difference between upper and lower joint tails is significant.
\end{abstract}

Keywords: Conditional Dependence; Tail Dependence; Copulas; Contagion

JEL: C22, G12 


\section{Introduction}

Modelling correlations and linkages between financial asset returns are important for international investors to diversify risk, and also for monetary policy makers to control for the risk of financial contagion. There are many studies on modelling the dynamic linkages between international stock markets and most have concluded the existence of market linkages and financial contagion (see, for example, Yiu et al., 2010; Kenourgios et al., 2011; Wen et al., 2012; Hui and Chan, 2013). However, only a few studies have modelled the tail structure of the dependence between markets. Furthermore, due to the recent turmoil, one should expect such dependence to be asymmetric, as comovements between markets will be affected by negative shocks more substantially than positive shocks. Relatively, sufficient evidence is found for the existence of asymmetric dependence between stock market returns. Cappiello et al. (2006) found evidence of asymmetry in conditional volatility of the equality returns. Tamakoshi and Hamori (2012) amongst others found the stock return comovements are more significantly influenced by negative shocks. However, these studies emphasized developed markets in Euro areas and the United States. Only a few have modelled the increased integration between China and its regional developed contender.

This paper aims to provide models for the entire dependence structure as well as asymmetric behaviour in bivariate tails for two Chinese stock indices using conditional copula models. The capitalisation of the Chinese stock market overtook Japan and became the world's second largest stock market, totalling $\$ 3,981$ billion in A-share capitalisation at the end of 2010 . The unique position of the Chinese stock market amongst its international competitors and its role during the recent financial crisis, has encouraged the study of its dependence with other mature financial markets during the period of turmoil.

The economic literature that focuses on the dynamic dependence between Chinese stock markets is somewhat dated. Li (2007) employed a multivariate GARCH model to test linkages in volatility between Shanghai, Shenzhen, Hong Kong and the US stock market using daily data from 2001 to 2005. Zhang (2009) examined the volatility spillover between Shanghai and Hong Kong stock markets from 1996 to 2008 by a Multivariate GARCH model while the linkages in returns are modelled using copulas. $\mathrm{Hu}$ (2010) found much lower tail dependence between Chinese and other international stock markets than the United States. Lai and Tseng (2010) modelled the dependence structure between China and the G7 stock markets using a mixture copula. However, their analysis is again based on a more stable period of the stock markets in China.

Therefore, we extend the literature by investigating the conditional dependence between the Chinese stock markets for a more turbulent period. In particular, we are interested in the relationship between Shanghai and Hong Kong stock markets, since studying the dynamics of the dependence between the two markets may provide interesting insights, as each of the markets has different features, but each was affected by the 07-09 subprime crisis and survived the financial turmoil so far. 
Since reaching an historical peak of 6,124 points on Oct 16,2007 , the Shanghai Stock Exchange Composite index suffered a record of 65\% drop in 2008, as a result of the global economic recession. Meanwhile, the Hong Kong Hang Seng stock index is more mature and internationalised. While stocks listed in the Hong Kong market can be traded freely amongst international investors, only a small amount of listed stocks in the Shanghai stock market (namely B-share stocks) can be invested in with foreign capital. In the meantime, the Hang Seng stock index is closely linked to the economy in Mainland China as most reference entities listed in this index has investment relations with Mainland China. This creates some particular distinguishing features as well as associations for these two stock indices during the financial crisis.

To assess the changing dependence structure, we implement a timevarying copula approach, where the generalised autoregressive score (GAS) model of Creal, et al. (2013) is used to capture the evolution of the copula parameters. Moreover, this paper extends the analysis of the dependence structure between Shanghai and Hong Kong stock markets in several other ways. First, we define the conditional margins separately for the two stock indices and introduce the methods of selecting the optimal model. Second, we provide the entire structure of dependence as well as the structure of bivariate tails. While constructing the tail dependence, we model the upper and lower tails separately, to capture the asymmetric property. Finally, tests for the time-varying structure and asymmetric dependence are introduced.

Our results suggest that the conditional dependence between the two markets is strongly time-varying. While the correlation decreased before the crisis, it increased significantly prior to 2008, suggesting the existence of contagion between the two markets. This is constant with Forbes and Rigobon (2002)'s standard definition of financial contagion that contagion exists if crossmarket co-movement increases significantly after a shock. The correlations persisted at a high level after 2008, which can be explained by herding behaviour as the crisis grew. Moreover, we find a slightly stronger bivariate upper tail, which has not been documented in previous literature, indicating asymmetric dependence. Testing for asymmetry shows that the difference between upper and lower joint tails is statistically significant.

Our results may provide investors and policy makers more timely information with regards to portfolio management, risk diversification, and asset allocation. While we show that the Chinese markets are less dependent before crisis but more dependent during the crisis, it may suggest market upturns in Hong Kong were mainly driven by the stock markets in Mainland China, while the bull markets were brought about by the global economic recession, this finding is also consistent with the asymmetric tail dependence found between Shanghai and Hong Kong stock markets.

The rest of the paper is organised as follows. Section 2 will discuss the models for the marginal distribution and the joint distribution of copulas. Section 3 will select the optimal models for the marginal distributions. Section 4 and 5 will build models for the entire dependence structure as well as for the bivariate tails. Section 6 concludes the paper. 


\section{Methodology}

We use copula to model the asymmetric conditional dependence between Shanghai and Hong Kong stock markets. A copula is a function that links marginal distributions of random variables to form a joint distribution. In other words, the joint distribution can be written as a copula function and marginal distributions. Thus, copula-based models provide great flexibility to model multivariate distributions, allowing for specification of the marginal distributions separately from the dependence structure (copula).

Since this paper focuses on the conditional dependence, we are interested in applying a version of Sklar's theorem (1959) as presented in Patton (2006). Assume the joint conditional distribution of random variables $R_{i t}$ is $H\left(r_{i t} \mid \Pi_{t-1}\right)$, where $i=1, \ldots, n$ and $\Pi_{t-1}$ is some information set. By decomposing it into $n$ marginal distributions, we have

$$
H\left(r_{i t} \mid \Pi_{t-1}\right)=C\left(F_{1}\left(r_{1 t} \mid \Pi_{t-1}\right), \ldots, F_{n}\left(r_{n t} \mid \Pi_{t-1}\right) \mid \Pi_{t-1}\right)
$$

The above function highlights the flexibility of copula-based models in estimation and model specification. That is, we can estimate the model in stages by first estimating the conditional marginal distributions and then consider copula models for the conditional joint distribution. This generates an $n$ dimensional model, without the challenge of estimating it simultaneously.

\subsection{Models for the Conditional Marginal Distribution}

Since we aim to construct the conditional dependence structure, we must first model the conditional margins. In this paper, we will allow the index return series to have time-varying conditional means and variances based on the following structure:

$$
R_{i t}=\mu_{i}\left(\phi_{t-1}\right)+\sigma_{i}\left(\phi_{t-1}\right) \varepsilon_{i t}
$$

where $i=1, \ldots, n, \phi_{t-1} \in \Pi_{t-1}$, and $\varepsilon_{i t}$ is the standardised residuals, which has the following expression:

$$
\varepsilon_{i t}=\frac{R_{i t}-\mu_{i}\left(\phi_{t-1} ; \theta\right)}{\sigma_{i}\left(\phi_{t-1} ; \theta\right)}, i=1, \ldots, n
$$

where $\theta$ is the vector of estimated parameters for the models of conditional mean and variance.

The conditional marginal distributions are calculated using the above standardised residuals. We will adapt a parametric model for conditional margins where the skewed $t$ of Hansen (1994) is selected for the distribution of standardised residuals. The skewed $t$ distribution is close to a $t$ distribution, but has an additional parameter to capture the asymmetry in distribution while maintaining a zero mean and unit variance. The skewed $t$ distribution is defined as:

$$
d(z \mid \eta, \lambda)= \begin{cases}b c\left(1+\frac{1}{\eta-2}\left(\frac{b z+a}{1-\lambda}\right)^{2}\right)^{-\frac{\eta+1}{2}} & \text { if } z<-a / b \\ b c\left(1+\frac{1}{\eta-2}\left(\frac{b z+a}{1+\lambda}\right)^{2}\right)^{-\frac{\eta+1}{2}} & \text { if } z \geq-a / b\end{cases}
$$


where $a \equiv 4 \lambda c \frac{\eta-2}{\eta-1}, b \equiv 1+3 \lambda^{2}-a^{2}, c \equiv \frac{\Gamma\left(\frac{\eta+1}{2}\right)}{\sqrt{\pi(\eta-2)} \Gamma\left(\frac{\eta}{2}\right)} . \eta$ and $\lambda$ denote the degree of freedom and the asymmetry parameter and $2<\eta<\infty$ and $-1<\lambda<1$. From the above model, we know that if $\lambda=0$, then the skewed $t$ distribution is a standardised student's $t$ distribution; if $\lambda>0$, then the density of the distribution is skewed to the right, and vice versa.

\subsection{Copula Models for the Entire Dependence Structure}

After modelling the conditional marginal distributions of the asset returns, we will obtain $n$ pairs of uniformly distributed variables $u_{i t}, i=1, \ldots, n$, which will be used to estimate the copula parameter.

In this paper, we will provide models for constant copulas as well as timevarying copulas. In particular, the time-varying copula will be modelled using the generalised autoregressive score (GAS) model of Creal, et al. (2013), which assumes the time-varying copula parameters follow an evolution function of the lagged copula parameter and a "forcing variable" that is related to the scale score of the copula log-likelihood.

The GAS (1,1) specification of Creal et al. (2013) will be implemented which has the following expression:

$$
\phi_{t+1}=\omega+\alpha \frac{2}{1-\rho_{t}^{2}}\left[A_{t}-\rho_{t}-\rho_{t} \frac{B_{t}-2}{1+\rho_{t}^{2}}\right]+\beta \phi_{t}
$$

where $\phi_{t}$ is the copula parameter, $\rho_{t}$ is the correlation parameter, $A_{t}=$ $\Phi^{-1}\left(u_{1 t}\right) \Phi^{-1}\left(u_{2 t}\right), B_{t}=\Phi^{-1}\left(u_{1 t}\right)^{2}+\Phi^{-1}\left(u_{2 t}\right)^{2}$ and $\Phi^{-1}(\cdot)$ is the inverse of the normal distribution function.

\subsection{Copula Models for the Tail Dependence}

One of the most important features of the structure of the relationship is the tail dependence. We consider this by first looking at the quantile dependence, which is captured by the following functions:

$$
\begin{aligned}
\lambda^{t} & =\left\{\begin{array}{l}
\operatorname{Pr}\left(U_{x} \leq t \mid U_{y} \leq t\right), 0<t \leq \frac{1}{2} \\
\operatorname{Pr}\left(U_{x}>t \mid U_{y}>t\right), \frac{1}{2}<t<1
\end{array}\right. \\
& =\left\{\begin{array}{c}
\frac{C(t, t)}{t}, 0<t \leq 1 / 2 \\
\frac{1-2 t+C(t, t)}{1-t}, 1 / 2<t<1
\end{array}\right.
\end{aligned}
$$

The above function provides a richer description of the dependence structure between two random variables. As $q \rightarrow 0$, we have the lower (left) tail dependence, which is defined as

$$
\lambda^{L}=\lim _{t \rightarrow 0^{+}} \frac{C(t, t)}{t}
$$

Similarly, the upper (right) tail dependence is defined as

$$
\lambda^{U}=\lim _{t \rightarrow 1^{-}} \frac{1-2 t+C(t, t)}{1-t}
$$

Therefore, as $t$ move from the centre (when $t=1 / 2$ ) to the tails, by comparing the lower/left $(t<1 / 2)$ and upper/right $(t>1 / 2)$ tails, it provides 
information on the dependence structure such as asymmetric dependence. This is extremely useful in cases that an asymmetric dependence is present, as many of the copula functions, such as Gaussian copula or student's $t$ copula, which capture only the symmetric dependence.

\section{Data}

This paper investigates the asymmetric conditional dependence structure between two Chinese stock index returns. Daily time-series data from 01 July 2003 to 9 February 2017 is obtained from Bloomberg for Shanghai Composite Stock Exchange index and Hong Kong Hang Seng Stock Exchange index. By removing non-trading dates in the sample period, we have in total 3314 observations. The log return is used which has the following expression:

$$
R_{t}=\ln \left(P_{t} / P_{t-1}\right)
$$

The dataset covers the recent crisis period, thus allows us to provide a comprehensive analysis of the dependence structure for the turmoil period. The descriptive statistics of the two index returns are presented in Table 1. As can be seen, both series have mean zero and very close variances. Both series have negative skewness and large positive kurtosis. However, the Shanghai stock index has a more negative skewness value, indicating more significant left tail risk. The Jarque-Bera again confirms the non-normality of both series. The linear and Spearman's rank correlation values indicate a strong positive correlation between the two returns.

The time-series plots of the index prices are present in Figure 1. We can see that the term structures of the two indices are quite similar, which indicates the existence of strong comovements between the two markets. Both index prices increased prior to the subprime crisis, and reached their peak during the crisis. Followed by significant price drop in 2008, both series were adjusted to a level higher than the pre-crisis period. While the Hang Seng index price continued to fluctuate around that level, the Shanghai index price was moving slowly towards a downward sloping direction after 2010 and rise significantly after 2014 .

\section{Model Selection}

\subsection{Conditional Mean}

To model the conditional dependence structure of the two financial index returns, we first estimate the conditional margins based on Equation (2). That is, we assume the return series has time-varying conditional means and variance. Moreover, we assume that the standardised residual $\varepsilon_{i t}$ to have a constant conditional distribution. Therefore, we first consider Autoregressive-Movingaverage (ARMA) models for the conditional mean of up to order $(5,5)$, and select the optimal model by applying the Bayesian Information Criterion (BIC). We find that AR(0) seems to be the optimal model for both Shanghai and Hong Kong stock index returns. The Ljung-Box test on residuals suggests no remaining autocorrelation from the optimal model. We also apply an F-test for the a significance of across-variable lags up to order 5 . The results suggest that there is significant across-variable relation from Shanghai to Hong Kong, but not vice- 
versa. This implies that the Shanghai Stock market leads the Hong Kong market and is more important in the price discovery process. The $t$-statistics shows that the Hong Kong stock index return is significantly influenced by the performance of the Shanghai Stock market from the previous day. Therefore, we build the following models for the conditional mean:

$$
\begin{gathered}
R_{S H, t}=c_{1}+\varepsilon_{1 t} \\
R_{H K, t}=c_{2}+\varphi_{1} R_{S H, t-1}+\varepsilon_{2 t}
\end{gathered}
$$

\subsection{Conditional Variance}

We consider the Glosten-Jagannathan-Runkle-generalized autoregressive conditional heteroscedasticity (GJR-GARCH) model of Glosten, et al. (1993) for the conditional variance. Models we have tested include the constant volatility model, ARCH(1), GARCH(1,1), GJR-GARCH(1,1,1), AR(2), GARCH(2,2) and GJR-GARCH $(2,2,2)$. The general GJR-GARCH class models have the following expression:

$$
\mathrm{h}_{\mathrm{t}}=\alpha_{0}+\sum_{i=1}^{n} \beta_{\mathrm{i}} \mathrm{h}_{\mathrm{t}-\mathrm{i}}+\sum_{i=1}^{n} \alpha_{\mathrm{i}} \varepsilon_{\mathrm{t}-\mathrm{i}}^{2}+\sum_{i=1}^{n} \alpha_{\mathrm{i}}^{*} \varepsilon_{\mathrm{t}-\mathrm{i}}^{2} \mathrm{I}_{\mathrm{t}-\mathrm{i}}
$$

where $\mathrm{I}_{\mathrm{t}-\mathrm{i}}=\left\{\begin{array}{l}1 \text { if } \varepsilon_{\mathrm{t}-\mathrm{i}}<0 \\ 0 \text { if } \varepsilon_{\mathrm{t}-\mathrm{i}} \geq 0\end{array}\right.$. We present the mean log-likelihood estimates and the BIC value for the above seven models in Table 2. As can be seen, BIC-optimal models for the Shanghai composite index return and the Hong Kong Hang Seng index return are both GJR-GARCH $(1,1,1)$, which has the lowest BIC value compare to other models.

The model parameters for time-varying means and variance from GJRGARCH $(1,1,1)$ specification are presented in Table 3. The GJR parameter for Shanghai and Hang Seng index returns are 0.003 and 0.173 , indicating that the negative error terms have a stronger effect on the future value of volatility for both stock indices.

The standardised residuals are then calculated from the optimal conditional variance models using Equation (3). Figure 2 plots the daily returns and standardised residuals for the Shanghai and Hang Seng stock indices. As can be seen, the existence of upper and lower tail dependence is evident, which indicates an extremely positive (negative) event in one market can be associated with an extremely positive (negative) event in the other market. The scatter plot for the standardised residuals shows there is only slight asymmetry between positive and negative standardised returns, which suggests that both returns responds to shocks similarly regardless whether the markets are booming or crashing during the sample period.

\subsection{Modelling Standardised Residuals}

In this paper, the marginal distribution is modelled using a parametric approach by the method of Inference Function for Margins (IFM). By assuming the random variable follows a specific cumulative distribution function, the IFM method transforms the random variable into a uniformly distributed variable, using the probability integral transformation function. 
In order to avoid the misspecification problem that is commonly found in a parametric approach, we first test a series of distributions for the standardised residuals, including Gaussian, Student's $t$, Generalised Error Distribution (GED) and skewed $t$ distribution. We then apply a Kolmogorov-Smirnov test to check which distribution is most close to the real distribution of the standardised residuals. The results show that the skewed $t$ distribution has the lowest $p$-value and hence is the most appropriate distribution for the model. The two parameter estimates of Skewed $t$ are presented in Table 3. As can be seen, both residual series have a negative skewness parameter, which suggests a left skewed distribution. This is consistent with the general economic condition, as there might be more adverse shocks than positive shocks during the crisis.

One of the disadvantages of the IFM method, as pointed by Chen and Fan (2006), is that the dependence parameters could be affected by a possibly misspecified marginal distribution of standardised innovations. Therefore, we further plot the fitted parametric estimates of the skewed $t$ distribution with the histogram of its empirical approximation in Figure 3. As noted, the fitted density of skewed $t$ is able to provide reliable estimates of the empirical histogram. The right panel of Figure 3 provides the quantile-quantile (Q-Q) plot for skewed $t$ distribution. As can be seen, there exist a few extreme left tail observations that are not captured by the models for the Shanghai Stock Exchange Composite index and Hang Seng Stock Index.

Finally, we apply a goodness-of-fit test on skewed $t$ distribution using a Kolmogorov-Smirnov (KS) test and a Cramer-von Mises (CvM) test, as introduced in Patton (2013), to check the goodness-of-fit of the fitted distribution. The two test statistics are

$$
\begin{gathered}
K S_{i}=\max _{t}\left|\hat{u}_{i,(t)}-t / T\right| \\
C v M_{i}=\sum_{t=1}^{T}\left(\hat{u}_{i,(t)}-t / T\right)^{2}
\end{gathered}
$$

where $\hat{u}_{i,(t)}$ is the $t^{t h}$ largest value of $\left\{\hat{u}_{i, j}\right\}_{j=1}^{T} \cdot u_{i t}$ is the probability integral transformations of the standardised residuals based on Hansen's skewed $t$ distribution. KS (CvM) test statistics on the two residual series are reported as $0.0189(0.2882)$ and $0.0231(0.3484)$. We then use a simulation-based method as introduced in Genest and Rémillard (2008) to calculate the $p$-value for both tests. The test statistics and the corresponding $p$-values are reported in Table 3 . Both tests suggest the rejection of the null that the skewed $t$ distribution is a wellspecified distribution for the standardised residuals.

\section{Dependence Structure between Shanghai and Hong Kong Stock Markets}

A common measure of dependence between two random variables is the linear and rank correlation coefficients, which have been reported in Table 1. However, linear correlation is not invariant under monotonic transformation. While rank correlation is an essential scalar measure for the sign and degree of dependency, they give no information on the structure of the relationship between two 
variables. Therefore, we will use copula to model the dependence structure between Shanghai and Hong Kong stock markets.

\subsection{Conditional Copula Estimates}

We now move on to model the conditional joint dependence using the probability integral transformation $u_{S H, t}$ and $u_{H K, t}$ of the standardised residuals. In this section, we will consider both constant and time-varying copulas. Estimation results of constant copula parameters are presented in Table 4. We estimate the copula parameters, the lower and upper tail dependence indicated by each copula and the value of the log-likelihood for seven constant copula functions, including the Normal, Student's $t$, Symmetrised Joe-Clayton (SJC), Gumbel, Clayton, Rotated Gumbel and Rotated Clayton copulas. Each copula function has unique features and different abilities to capture the tail dependence. ${ }^{1}$ The results suggest that the student's $t$ copula is the best fit for the entire dependence structure, followed by the SJC and Normal copulas, as the student's $t$ copula has the lowest negative loglikelihood value. $^{2}$

The tail dependence reported in Table 4 reveals some interesting features on these copula models. As can be seen, the student's $t$ copula has symmetric lower and upper tail dependence. The tail dependence of the student's $t$ copula is slightly larger than its linear correlation parameters, suggesting that the Shanghai and Hong Kong stock markets are more dependent under extreme events. The SJC copula has larger lower tail dependence, indicating the existence of asymmetric dependence. As suggested by Table 4, rotated Clayton copula has the lowest loglikelihood value and hence fails to capture the dependence structure. This might related to the zero lower tail dependence imposed by rotated Clayton copula, which might be an incorrect reflection of the general dependence feature during the crisis. We also investigate whether the long-run relationships are not constant over time.

\subsection{Testing for Time-varying Correlation}

Since we aim to model the conditional dependence structure, it would be more realistic to consider the dependence between two financial assets changing through time. In fact, there are extensive literatures that already account for the time-varying nature of the conditional volatility of financial assets. One of the fundamental methods in this field is the dynamic conditional correlation (DCC) proposed by Engle (2002). Under a DCC framework, the covariance matrix is defined as

$$
H_{t}=D_{t} R_{t} D_{t}
$$

\footnotetext{
${ }^{1}$ For more details on these copulas, please refer to Joe (1997, Chapter 5) or Nelsen (2006, Chapter 4-5).

2 It should be noted that, due to the large sample size when calculating the information criteria (e.g. AIC or BIC) for these copula models, the number of copula parameters does not affect the final results of the goodness-of-fit test.
} 
where $D_{t}=\left[\begin{array}{cc}\sqrt{h_{11 t}} & 0 \\ 0 & \sqrt{h_{22 t}}\end{array}\right], R_{t}=\left[\begin{array}{cc}1 & \rho_{12} \\ \rho_{21} & 1\end{array}\right]$ and the conditional correlation $R_{t}$ is given as

$$
R_{t}=\left(\operatorname{diag} Q_{t}\right)^{-1 / 2} Q_{t}\left(\operatorname{diag} Q_{t}\right)^{-1 / 2}
$$

where the positive symmetric matrix $Q_{t}$ is given as

$$
Q_{t}=(1-a-b) \Psi+a \varepsilon_{t-1} \varepsilon_{t-1}^{\prime}+b Q_{t-1}
$$

where $\varepsilon_{i t}=e_{i t} / \sqrt{h_{i i t}}$ is the standardised residuals, $\Psi$ is the $\mathrm{N} \times \mathrm{N}$ unconditional correlation matrix of $\varepsilon_{t}$. Both $\mathrm{a}$ is positive, and $\mathrm{b}$ is non-negative scalar parameters satisfying $a+b<1$.

Table 5 presents the estimation results of the DCC model of Eagle (2002), using the same specifications for conditional means and variance as described earlier. Our results reveal a large degree of persistence in the conditional correlation process, with $\theta_{1}+\theta_{2}$ very close to one. As suggested by Hsu et al. (2008), a high degree of persistence in dynamic conditional correlation means crashes can push the correlations away from its long-run mean and the correlations will have more volatile responses to new information.

Figure 4 plots the 60-day rolling window rank correlations and the dynamic conditional correlations between the Shanghai and Hong Kong stock index returns. As can be seen, both time-varying correlations increased since 2006 while reaching the peak in early 2008. As stated by Forbes and Rigobon (2002), contagion happens when the cross-market correlation dramatically increases after a shock. Therefore, this huge increase in correlation may point to the existence of financial contagion between the two markets. The correlation coefficients keep fluctuating at a higher level between 0.5 and 0.6 after 2008, indicating a consistent higher correlation during and after the crisis period. This is an interesting finding, as earlier research such as Zhu (2001) shows that stock markets in Mainland China are completely independent and not affected by outlier stock markets. The constant higher correlation after 2008 may be explained by herding behaviour as the crisis grew.

However, using the DCC model for the conditional correction restricts the distribution of the standardised residuals to an elliptical distribution. Hence, we consider using the time-varying copula approach to capture the changing dependence. Before modelling the time-varying copulas, we first introduce an Autoregressive Conditional Heteroskedasticity Lagrange Multiplier (ARCH LM), which looks for autocorrelation as a measure of dependence, to check the existence of time-varying dependence. The test is based on the following autoregression model:

$$
S_{S H, t} S_{H S, t}=\alpha_{0}+\sum_{i=1}^{q} \alpha_{1} S_{S H, t-i} S_{H S, t-i}+\varepsilon_{t}
$$

where $S_{t}$ is the standardised residual from the CCC-GARCH model with the same mean and variance specifications for Shanghai and Hang Seng index returns. Under the null hypothesis of a constant conditional copula, we should have $\alpha_{i}=0$ for $i=1, \ldots, q$. The test statistics and $p$-values are presented in the lower panel of Table 5. As can be seen, the null that a constant conditional correlation is rejected for all cases. Therefore, we can conclude that there is strong evidence against 
constant conditional correlation and thus evidence against a constant conditional copula.

\subsection{Time-varying Dependence Structure Captured by Copula}

Patton (2006) proposed the time-varying copula model based on assumptions that the current dependence parameter is associated with the past dependence parameter and the historical mean of difference of cumulative probability integral transformations. Creal, et al. (2013) proposed a Generalised Autoregressive Score (GAS) model, which extends Patton (2006)'s work on modelling time-varying copulas.

In this paper, the time-varying copula parameter is modelled by Creal, et al. (2013)'s GAS model as described in Equation (5). Three time-varying copulas are estimated: the time-varying Gaussian copula, the time-varying student's $t$ copula and the time-varying rotated Gumbel copula. The corresponding parameters are presented in Table 4 . As can be seen, the time-varying student's $t$ copula performs better in describing the dependence structure due to the lowest negative log-likelihood value, which may point to the fact that heavy tail dependence exists between the Shanghai and Hong Kong stock markets.

After estimating the time-varying copula parameters, we introduce a simulation approximation as described in Patton (2013) to plot the time-varying linear correlation as indicated by the student's $t$ and Gumbel copulas. We are extremely interested in observing the time-varying nature of the above two copulas since both copulas account for the tail dependence. The Gumbel copula parameter is required to be in the range $(1, \infty)$, therefore, the correlation parameter is modelled by $\rho_{t}=1+\exp \left(\phi_{t}\right)$ to ensure this, while for the student's $t$ copula, the correlation parameter is modelled by $\rho_{t}=\left[1-\exp \left(-\phi_{t}\right)\right] /[1+$ $\left.\exp \left(-\phi_{t}\right)\right]$

The linear correlations indicated by the time-varying student's $t$ and Gumbel copulas are plotted in Figure 5. Compared to the time-varying rank correlations and the DCCs, we find some additional changes with regards to the dependence structure. There is a significant decrease in correlation between the two markets before the crisis. The correlation coefficient dropped from 0.5 to 0.3 from 2006 to 2008, which shows that the Chinese stock market was relatively independent of other Asia financial markets such as the Hong Kong stock market before the crisis. This may have some important implications to portfolio management as investors could add the Mainland Chinese stocks to their portfolio to diversify risk.

This temporary decrease in correlation is decided by the opposite movements and unique features of the two markets. While the Hong Kong stock market is completely open to foreign investors, with its price dynamics more linked to the global economy, the movement of Shanghai stock index is mainly driven by domestic factors. This is because only limited stocks (called B-share stocks) listed in the Shanghai stock exchange are open to foreign capital. This directly affects the correlation between the two indices before the crisis, as the 
stock markets in Mainland China were experiencing its greatest boom, with index prices rising from 1,200 points to over 5,000 points in early 2008 .

However, this negative relation reversed as a direct effect of the crisis, causing both markets to move downwards. Contagion effect was observed since the beginning of 2008, with signs of significant increases in cross-market correlations between the two markets. The correlations persisted at a high level after 2009, which can be explained by herding behaviour as the crisis grew. The increasing correlation since the start of the crisis reveals an enhancing integration between Chinese stock markets and regional developed markets. Thus, we might expect the dependent increase in future as Chinese stock markets becoming more open to foreign capital. A program that aims to promote two-way opening-up of the capital market on the mainland and Hong Kong was officially launched on 17 November 2014 by the Chinese Premier Li Keqiang. This "Shanghai-Hong Kong Stock Connect" initiative allows foreign investors to access 568 Shanghai stocks through the Hong Kong Stock Exchange. Conversely, Chinese investors will be able to trade Hong Kong's stocks, known as H-shares, through the system.

Our results on modelling the time-varying correlation may provide international investors more timely information with regards to portfolio management, risk diversification, and asset allocation. While we show that the two stock markets are less dependent before the crisis but more dependent during the crisis it may suggest that the market upturns in Hong Kong were mainly driven by the stock markets in Mainland China, while the bull markets were brought about by the global economic recession.

\section{Tail Dependence between Shanghai and Hong Kong Stock Markets}

Tail dependence is an important feature for financial asset returns as it reveals the comovements between assets under extreme conditions. Therefore, analysis of tail dependence is crucial, as both return series would experience extreme fluctuations during the crisis period.

\subsection{Measuring Tail Dependence using Copula}

Tail dependence can be measured as a function of copula as described in Equation (7) and (8). In this paper, we model the upper and lower bivariate tails separately in order to capture asymmetric dependence. The upper tail dependence will be estimated using a fully parametric approach by maximising the log-likelihood function as described in Chen, et al. (2010):

$$
L\left(\alpha_{i} \mid q\right)=\frac{1}{n} \sum_{t=1}^{n} \ell_{i}\left(\alpha_{i} \mid q\right)
$$

where $\ell_{i}\left(\alpha_{i} \mid q\right)=\delta_{1 t} \delta_{2 t} \log c_{i}\left(\tilde{u}_{1 t}, \tilde{u}_{2 t} ; \alpha_{i}\right)+\delta_{1 t}\left(1-\delta_{2 t}\right) \log \frac{\partial c_{i}\left(\widetilde{u}_{1 t}, \tilde{u}_{2 t} ; \alpha_{i}\right)}{\partial u_{1 t}}$

$$
+\left(1-\delta_{1 t}\right) \delta_{2 t} \log \frac{\partial c_{i}\left(\tilde{u}_{1 t}, \tilde{u}_{2 t} ; \alpha_{i}\right)}{\partial u_{2 t}}+\left(1-\delta_{1 t}\right)\left(1-\delta_{2 t}\right) \log c_{i}\left(\tilde{u}_{1 t}, \tilde{u}_{2 t} ; \alpha_{i}\right)
$$

and $\tilde{u}_{1 t}=\max \left[u_{1 t}, \alpha_{i}\right], \tilde{u}_{2 t}=\max \left[u_{2 t}, \alpha_{i}\right], \delta_{1 t}=1\left\{u_{1 t}>q\right\}, \delta_{2 t}=1\left\{u_{2 t}>q\right\}$. The lower tail will be estimated similarly under the condition $u_{1 t}<q, u_{1 t}<q$. After obtaining the optimal tail copula parameter $\alpha_{i}$ through maximum log- 
likelihood estimation (MLE), we can calculate the upper and lower tail dependence using Equation (7) and (8).

Using the estimator given by Frahm, et al. (2005), we calculate the tail dependence for the student's $t$ and Gumbel copulas. Compared to the student's $t$ copula, where both bivariate tails are estimated similarly, the tail dependence for the Gumbel copula is approximated separately using a Gumbel copula for the upper tail and a rotated Gumbel for the lower tail. The estimation results for the tail dependence are presented in Table 6. The upper and lower tail copula parameters for the Gumbel copula are 1.05 and 1.08 whereas for the student $t$ copula, the parameters are 6.02 and 0.20 respectively. Using the one-to-one mapping between the copula parameter $\alpha$ and the kendall's tau rank correlation

$$
\tau_{\text {Gumbel }}=\frac{\alpha-1}{\alpha}, \tau_{\text {student }}=\frac{2}{\pi} \arcsin (\alpha),
$$

we can obtain measures for the corresponding tail dependence.

As can be seen, tail dependence implied by the student's $t$ copula is lower, which implies that the Gumbel copula accounts for more extreme comovements than the student's $t$ copula. Tail dependence parameters are low for both copulas, indicating the probability that an extreme comovement between the Shanghai and Hang Seng stock indices is low.

Figure 6 plots the tail dependence captured by the Gumbel copula for different quantile $q$. We could see a clear increase in dependence as the quantile moving towards the centre of the distribution. Besides, evidence of asymmetric tail dependence can be observed. A much stronger bivariate upper tail is observed when the quantile $q$ approaches zero. However, the lower tail dependence becomes stronger as the quantile $q$ moving towards the centre. Moreover, immediate flat spot does not observed for both bivariate tails. There are noticeable fluctuations for upper tail dependence suggesting some cut-off when calculating the tail dependent parameters.

\subsection{Testing for Conditional Asymmetric Dependence}

After obtaining the upper and lower tail dependence parameters, we run tests for asymmetric dependence. Tests for conditional asymmetric dependence can provide important insights on whether two financial asset returns exhibit stronger correlation during market downturns. One way to see this is to run a simple test on the following null hypothesis:

$$
\lambda^{q}=\lambda^{1-q}
$$

where $\lambda$ is the quantile dependence. In this paper, we run the test jointly for a list of different $q$ values $0.025,0.05,0.10,0.975,0.95$, and 0.90 . Therefore, we redefine and tests the null hypothesis for

$$
\lambda^{q}=\left[\lambda^{0.025}, \lambda^{0.05}, \lambda^{0.10}, \lambda^{0.975}, \lambda^{0.95}, \lambda^{0.90}\right]^{\prime}
$$

$$
\Theta \lambda^{q}=0
$$

where $\Theta=[1,1,1,-1,-1,-1]^{\prime}$. This test simplifies the procedure of producing test statistics for each individual quantile and interpreting for multiple test results. The corresponding chi-squared statistics and $p$-values are presented in Table 6 for 
both student's $t$ and Gumbel copulas. The results are consistent over different copula models, with $p$-values 0.04 and 0.02 for the student's $t$ and Gumbel copulas. Thus we accept the null that the conditional dependence between Shanghai and Hong Kong stock index returns is symmetry.

Another way to see the asymmetric dependence is to run a test on bivariate tails instead of testing different quantiles. Thus, we test the following null hypothesis:

$$
\lambda^{\text {Lower }}=\lambda^{\text {Upper }}
$$

The lower and upper tail dependence parameters used in the test are calculated from Section 6.1. Significant differences between upper and lower tails are found between the upper and lower tails using the Gumbel copula and the student's $t$ copula. In conclusion, we have a slight stronger lower tail parameter, indicating asymmetric dependence, meaning that the two stock markets show more interdependence when in bear periods than during bull periods. Hence, there is a high probability of joint extreme events during the market downturns than during the market upturns.

\section{Conclusion}

This paper models the asymmetric dependence structure between Shanghai and Hong Kong stock index returns during 2003-17 using the copula approach. We extend the literature by first examining the dependence for a more turbulent period, when extreme events and tail movements were common. Then we construct the dependence structure using various copula models.

We find significant time-varying nature in correlation between Shanghai and Hong Kong stock markets. The correlation decreased before the crisis and increased significantly prior to 2008, suggesting the existence of financial contagion between the two markets. This increase however slowed down since 2009. The increasing correlation since the start of the crisis reveals an enhancing integration of the Chinese stock market with regional developed markets. Thus, we might expect the dependence to increase in the future as Chinese stock markets become more open to foreign capital.

Our studies also extend the literature of modelling dependence in the following ways: We define the conditional margins separately for the two stock indices and introduce methods of selecting the optimal model. Second, when constructing the tail dependence, we model the upper and lower tails separately, to capture the asymmetric property. Finally, tests for the time-varying structure and asymmetric dependence are introduced.

Our findings may provide more timely information with regards to portfolio management, risk diversification, and asset allocation. While we show that the Chinese market is less dependent before the crisis but more dependent during the crisis, it may suggest that the market upturns in Hong Kong were mainly driven by the stock markets in Mainland China, while the bull markets 
were brought about by the global economic recession, this finding is also consistent with the asymmetric tail dependence found between Shanghai and Hong Kong stock markets. 


\section{Reference}

Cappiello, L., Engle, R. and Sheppard, K. (2006) 'Asymmetric Dynamics in the Correlations of Global Equity and Bond Returns', Journal of Financial Econometrics, 4(4), 537-572.

Chen, X. and Fan, Y. (2006) 'Estimation of Copula-Based Semiparametric Time Series Models', Journal of Econometrics, 130(2), 307-335.

Chen, X., Fan, Y., Pouzo, D. and Ying, Z. (2010) 'Estimation and Model Selection of Semiparametric Multivariate Survival Functions under General Censorship', Journal of Econometrics, 157(1), 129-142.

Creal, D., Koopman, S. and Lucas, A. (2013) 'Generalized Autoregressive Score Models with Applications', Journal of Applied Econometrics, 28(5), 777795.

Engle, R. (2002) 'Dynamic Conditional Correlation', Journal of Business \& Economic Statistics, 20(3), 339-350.

Forbes, K. and Rigobon, R. (2002) 'No Contagion, Only Interdependence: Measuring Stock Market Comovements', The Journal of Finance, 57(5), 2223-2261.

Frahm, G., Junker, M. and Schmidt, R. (2005) 'Estimating the Tail-Dependence Coefficient: Properties and Pitfalls', Insurance: Mathematics and Economics, 37(1), 80-100.

Genest, C. and Remillard, B. (2008) 'Validity of the Parametric Bootstrap for Goodness-of-Fit Testing in Semiparametric Models', Annales de l'institut Henri Poincaré (B) Probabilités et Statistiques, 44, 1096-1127.

Glosten, L., Jagannathan, R. and Runkle, D. (1993) 'On the Relation between the Expected Value and the Volatility of the Nominal Excess Return on Stocks', The Journal of Finance, 48(5), 1779-1801.

Hansen, B. (1994) 'Autoregressive Conditional Density Estimation', International Economic Review, 35(3), 705-730.

Hsu, C., Tseng, C. and Wang, Y. (2008) 'Dynamic Hedging with Futures: A CopulaBased GARCH Model', Journal of Futures Markets, 28(11), 1095-1116.

Hu, J. (2010) 'Dependence Structures in Chinese and US Financial Markets: A Time-Varying Conditional Copula Approach', Applied Financial Economics, 20(7), 561-583.

Hui, E. and Chan, K. (2013) 'Contagion Across Real Estate and Equity Markets during European Sovereign Debt Crisis', International Journal of Strategic Property Management, 17(3), 305-316.

Joe, H. (1997) Multivariate Models and Dependence Concepts, Monographs on Statistics \& Applied Probability, Great Britain: Chapman \& Hall. 
Kenourgios, D., Samitas, A. and Paltalidis, N. (2011) 'Financial Crises and Stock Market Contagion in a Multivariate Time-Varying Asymmetric Framework', Journal of International Financial Markets, Institutions and Money, 21(1), 92-106.

Lai, Y. and Tseng, J. (2010) 'The Role of Chinese Stock Market in Global Stock Markets: A Safe Haven or a Hedge?', International Review of Economics \& Finance, 19(2), 211-218.

Li, H. (2007) 'International Linkages of the Chinese Stock Exchanges: A Multivariate GARCH Analysis', Applied Financial Economics, 17(4-6), 285297.

Nelsen, R. (2006) An Introduction to Copulas, Second Edition ed., New York: Springer Verlag.

Patton, A. (2006) 'Modelling Asymmetric Exchange Rate Dependence', International Economic Review, 47(2), 527-556.

Patton, A. (2013) 'Chapter 16 - Copula Methods for Forecasting Multivariate Time Series' in Graham, E. and Allan, T., eds., Handbook of Economic Forecasting, Elsevier, 899-960.

Sklar, A. (1959) 'Fonctions de Répartition à n Dimensions et Leurs Marges', Publications de l'Institut de Statistique de I'Université de Paris, 8(1), 229231.

Tamakoshi, G. and Hamori, S. (2012) 'An Asymmetric Dynamic Conditional Correlation Analysis of Linkages of European Financial Institutions during the Greek Sovereign Debt Crisis', The European Journal of Finance, 19(10), 939-950.

Wen, X., Wei, Y. and Huang, D. (2012) 'Measuring Contagion between Energy Market and Stock Market during Financial Crisis: A Copula Approach', Energy Economics, 34(5), 1435-1446.

Yiu, M., Ho, W. and Choi, D. (2010) 'Dynamic Correlation Analysis of Financial Contagion in Asian Markets in Global Financial Turmoil', Applied Financial Economics, 20(4), 345-354.

Zhang, S., Paya, I. and Peel, D. (2009) 'Linkages between Shanghai and Hong Kong Stock Indices', Applied Financial Economics, 19(23), 1847-1857. 
Table 1 Descriptive Statistics of the Index Returns

\begin{tabular}{lrr}
\hline & Shanghai & Hong Kong \\
\hline Minimum & -0.093 & -0.136 \\
$1^{\text {st }}$ Quartile & -0.007 & -0.006 \\
Mid & 0 & 0 \\
Mean & 0 & 0 \\
$3^{\text {rd }}$ Quartile & 0.009 & 0.007 \\
Maximum & 0.090 & 0.134 \\
\hline Std. Dev. & 0.017 & 0.015 \\
Skewness & -0.442 & -0.019 \\
Kurtosis & 7.097 & 13.091 \\
Jarque-Bera ( $p$-value) & 0 & 0 \\
Correlation (Linear|Rank) & 0.452 & 0.406 \\
\hline
\end{tabular}


Table 2 Comparisons of 7 GJR-GARCH Class Models for Conditional Variance

\begin{tabular}{lrrrr}
\hline & \multicolumn{2}{c}{ Shanghai } & \multicolumn{2}{c}{ Hong Kong } \\
\hline & Log-likelihood & BIC & Log-likelihood & BIC \\
Constant Volatility & 2.6486 & -5.2948 & 2.7745 & -5.5465 \\
ARCH(1) & 2.6742 & -5.3435 & 2.6485 & -5.2920 \\
GARCH $(1,1)$ & 2.7863 & -5.5627 & 2.9766 & -5.9459 \\
GJR-GARCH(1,1,1) & 2.7861 & -5.5649 & 2.9834 & -5.9496 \\
ARCH(2) & 2.7003 & -5.3933 & 2.6263 & -5.2453 \\
GARCH(2,2) & 2.7872 & -5.5622 & 2.9780 & -5.9438 \\
GJR-GARCH(2,2,2) & 2.7867 & -5.5562 & 2.9687 & -5.9275 \\
\hline
\end{tabular}


Table 3 Models for the Marginal Distribution

\begin{tabular}{lrr}
\hline & Shanghai & Hong Kong \\
\hline \multicolumn{1}{c}{$c$} & & 0 \\
\multicolumn{1}{c}{$\varphi_{1}$} & 0 & $-0.050^{* *}$ \\
Ljung-Box (5) & & 0.86 \\
Ljung-Box (10) & 0.13 & 0.72 \\
Chi^2 statistics & 0.20 & 10.033 \\
Chi^2 $p$-value & 4.879 & 0.074 \\
\hline Conditional Variance & 0.431 & \\
\hline Constant & & $0 * *$ \\
ARCH & $0.055^{* *}$ & $0.078^{* *}$ \\
GARCH & $0.939^{* *}$ & $0.764^{* *}$ \\
GJR & 0.003 & $0.173^{* *}$ \\
\hline Skewed $t$ & & \\
\hline Skewness & -0.0481 & -0.0537 \\
d.f. & 5.0580 & 6.3269 \\
KS test Stat. & 0.0189 & 0.0231 \\
( $p$-value) & $(0.07)$ & $(0.09)$ \\
CVM test stat. & 0.2882 & 0.3484 \\
( $p$-value) & $(0.11)$ & $(0.13)$ \\
$* *$ Significant at $5 \%$ level &
\end{tabular}


Table 4 Estimation Results for Copula Models

\begin{tabular}{|c|c|c|c|c|c|}
\hline \multirow[t]{3}{*}{ Constant } & \multirow{3}{*}{$\hat{\phi}$} & \multirow{3}{*}{$\hat{v}^{-1}$} & \multirow{2}{*}{\multicolumn{2}{|c|}{ Tail Dependence }} & \multirow{3}{*}{ Log-Likelihood } \\
\hline & & & & & \\
\hline & & & Lower & Upper & \\
\hline Student's $t$ & 0.4277 & 0.0765 & 0.6236 & 0.6236 & 353.0113 \\
\hline SJC & 0.2822 & 0.1676 & 0.2822 & 0.1676 & 351.0817 \\
\hline Normal & 0.4324 & & 0 & 0 & 343.0675 \\
\hline Rotated Gumbel & 1.3551 & & 0.3322 & 0 & 338.5495 \\
\hline Clayton & 0.5844 & & 0.3054 & 0 & 304.3771 \\
\hline Gumbel & 1.3334 & & 0 & 0.3182 & 288.5991 \\
\hline Rotated Clayton & 0.4994 & & 0 & 0.2496 & 224.0376 \\
\hline \multirow[t]{2}{*}{ Time-varying } & & & & & \\
\hline & $\hat{\phi}$ & $\hat{v}^{-1}$ & $\hat{\alpha}$ & $\hat{\beta}$ & Log-Likelihood \\
\hline TV-Student's $t$ & 0.0011 & 0.0351 & 0.0130 & 0.9990 & 410.9783 \\
\hline $\begin{array}{l}\text { TV-Rotated } \\
\text { Gumbel }\end{array}$ & -0.0009 & & 0.0204 & 0.999 & 382.1893 \\
\hline TV-Normal & 0.0024 & & 0.0962 & 2.0589 & 378.0085 \\
\hline
\end{tabular}


Table 5 Testing for Time-varying Dependence

\begin{tabular}{lcrrr}
\hline DCC Specification & \multicolumn{1}{c}{} \\
& $\rho$ & $\alpha$ & $\beta$ & Log-Likelihood \\
& 0.4350 & 0.0051 & 0.9938 & -3397.8642 \\
\hline \multicolumn{5}{l}{ Testing for Time-varying Dependence } \\
Test Statistics & AR(1) & AR(5) & AR(10) & AR(15) \\
$p$-value & 0 & 495.635 & 510.739 & 524.356 \\
\hline
\end{tabular}


Table 6 Tail Dependence between Shanghai and Hong Kong Stock Markets

\begin{tabular}{|c|c|c|c|}
\hline \multicolumn{4}{|c|}{ Tail Dependence } \\
\hline & & Upper Tail & Lower Tail \\
\hline \multirow[t]{2}{*}{ Gumbel } & $\tau$ & 0.0738 & 0.1049 \\
\hline & $\alpha$ & 1.0574 & 1.0842 \\
\hline \multirow{5}{*}{ Student's $t$} & $90 \% \mathrm{Cl}$ & [0.0356 0.1173$]$ & {$\left[\begin{array}{llllll}0.0600 & 0.1528\end{array}\right]$} \\
\hline & $\tau$ & 0.0465 & 0.0674 \\
\hline & $\alpha$ & 6.0187 & 0.2010 \\
\hline & $\lambda$ & 5.8794 & 15.3238 \\
\hline & $90 \% \mathrm{Cl}$ & {$\left[\begin{array}{lll}0.0315 & 0.1214\end{array}\right]$} & [0.0005 0.0781] \\
\hline \multicolumn{4}{|c|}{ Testing for Asymmetry } \\
\hline \multirow{3}{*}{ Difference } & & Gumbel & Student's $t$ \\
\hline & $\mathrm{Chi}^{\wedge} 2$ statistics & 8.3400 & 10.4700 \\
\hline & Chi^2 $p$-value & 0.0395 & 0.0150 \\
\hline \multirow[t]{2}{*}{ Equality } & test statistics & 1.9224 & 2.1261 \\
\hline & $p$-value & 0.0546 & 0.0335 \\
\hline
\end{tabular}

Note: lower tail of Gumbel is measured by the rotated Gumbel copula 
Figure 1 Shanghai Composite and Hang Seng Stock Index Prices

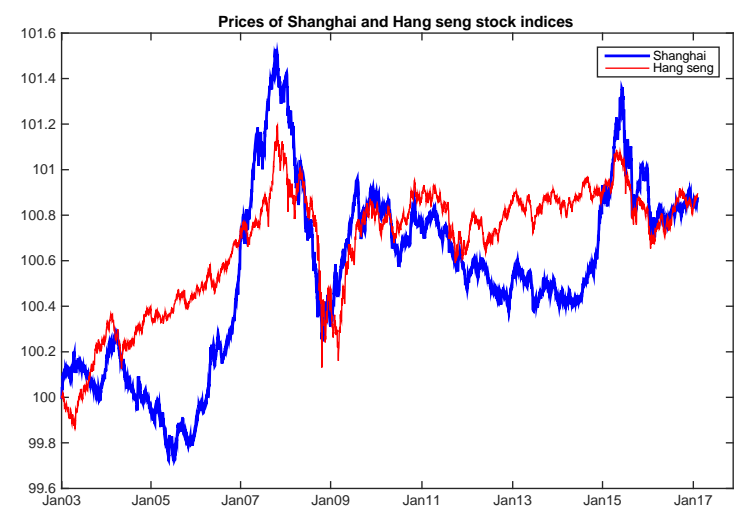


Figure 2 Daily Return and Standardised Residuals for Shanghai and Hang Seng Stock Indices
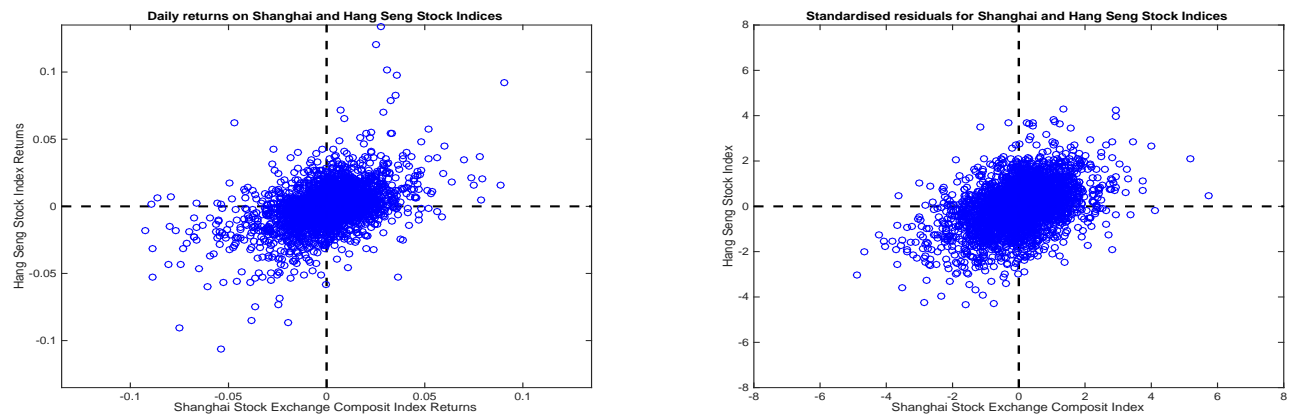
Figure 3 Skewed $t$ Distribution and the Q-Q plot
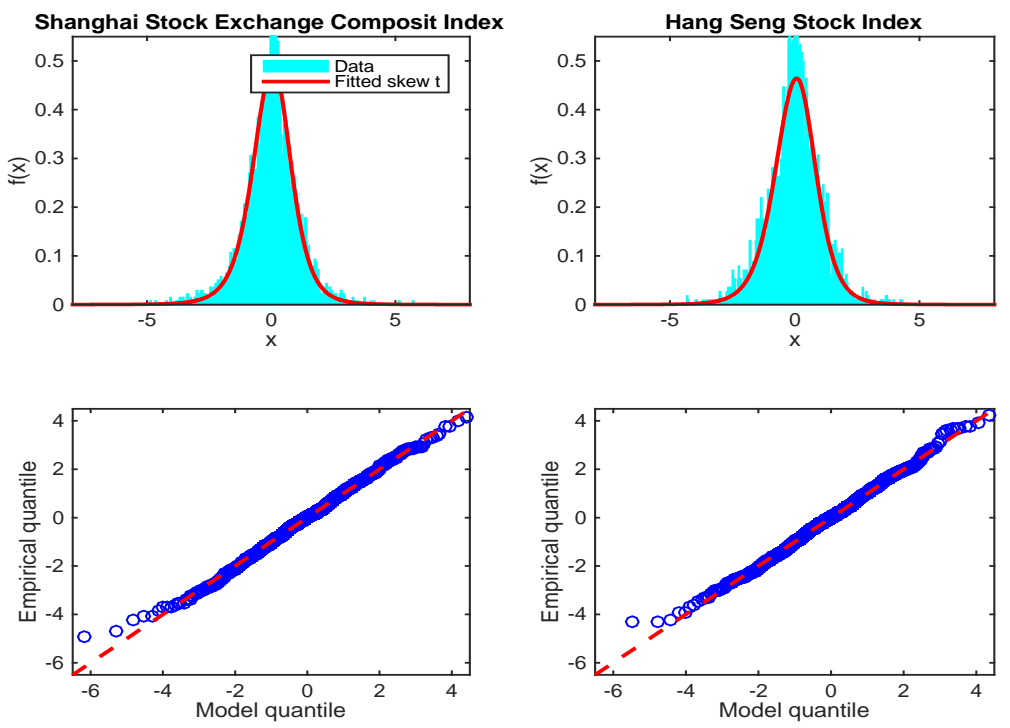
Figure 4 Time-varying Correlations between Standardised Returns of Shanghai and Hong Kong Stock Markets

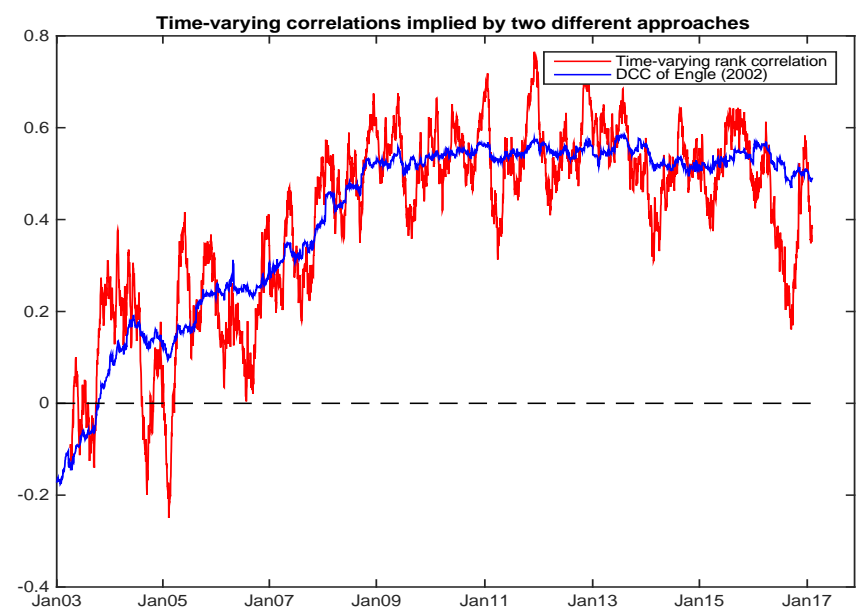


Figure 5 Linear Correlation Implied by Time-varying Copulas

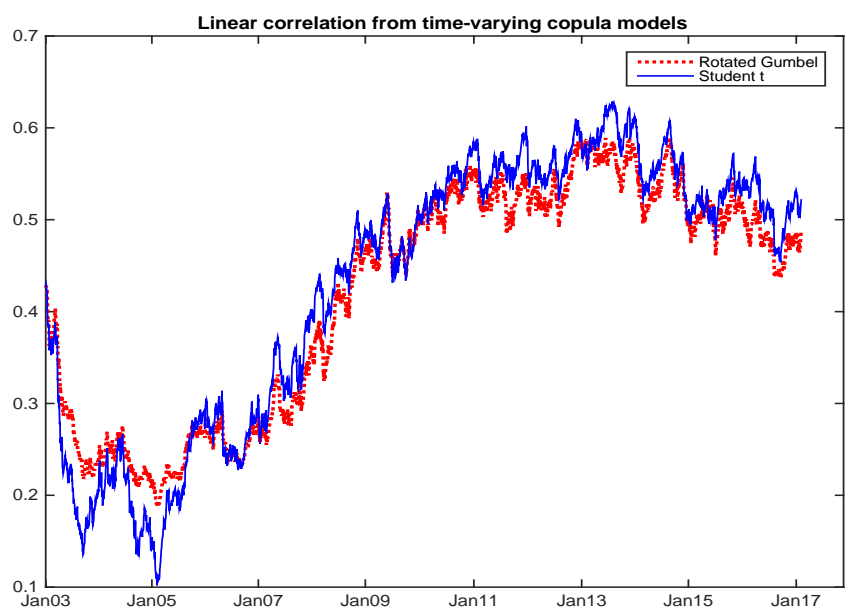


Figure 6 Tail Dependence Captured by Gumbel Copula

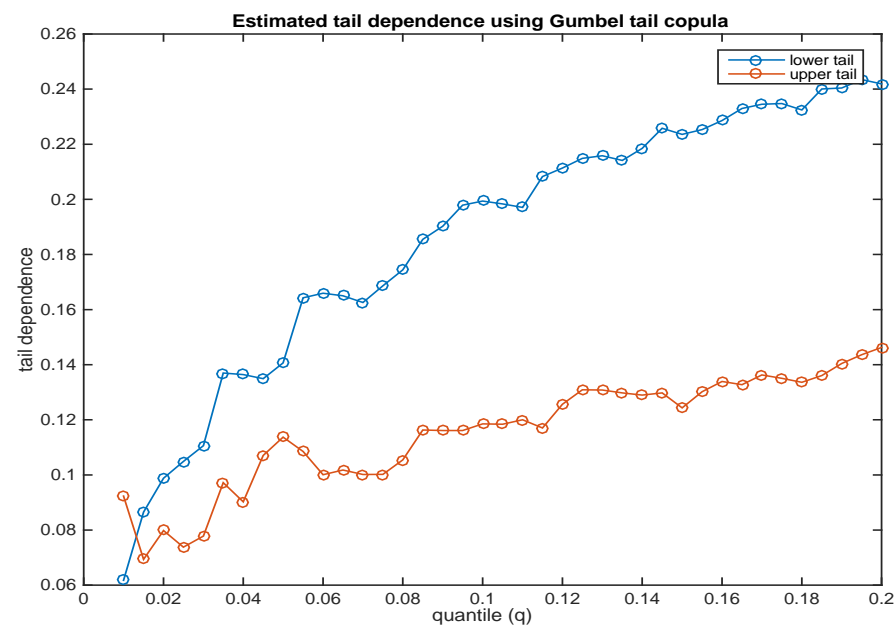

Note: The lower tail dependence is captured by the rotated Gumbel copula 